\title{
Magnitudes and Correlates of Human Immunodeficiency Virus, Hepatitis B Virus, and Syphilis among Pregnant Mothers Attending Antenatal Care in Addis Ababa, Ethiopia
}

\author{
Kassa Genetu $\mathbb{D}^{1},{ }^{1}$ Kerebih Abere $\mathbb{D}^{2},{ }^{2}$ and Erdaw Tachbele $\mathbb{D}^{2}$ \\ ${ }^{1}$ College of Health Sciences and Medicine, Dilla University, Dilla, Ethiopia \\ ${ }^{2}$ College of Health Sciences, Addis Ababa University, Addis Ababa, Ethiopia \\ Correspondence should be addressed to Erdaw Tachbele; erdawt@yahoo.com
}

Received 18 September 2021; Accepted 7 February 2022; Published 16 February 2022

Academic Editor: Atif Amin Baig

Copyright ( 2022 Kassa Genetu et al. This is an open access article distributed under the Creative Commons Attribution License, which permits unrestricted use, distribution, and reproduction in any medium, provided the original work is properly cited.

\begin{abstract}
Background. Human immunodeficiency virus (HIV), hepatitis B virus (HBV), and syphilis are major sexually transmitted infections (STIs) among the general population in Ethiopia, which in turn result in a wide range of adverse pregnancy outcomes. Hence, it is important to determine the seroprevalence and risk factors of HIV, HBV, and syphilis infection among pregnant mothers attending antenatal care in Addis Ababa, Ethiopia. Method. A cross-sectional study was conducted among 286 pregnant women from February 1, 2021, to March 30, 2021, in four selected public hospitals in Addis Ababa. Sociodemographic, risky sociocultural, behavioral, and hospital-related factors were collected using an interview-administered questionnaire. Detection of anti-HIV antibodies, hepatitis B surface antigen (HBsAg), and rapid plasma reagin (RPR) for syphilis was conducted. A binary logistic regression analysis was used to determine predictors of STIs using SPSS version 25. Result. A total of 281 pregnant mothers with a mean age of 27.5 (SD 4.6) completed the study. Among the participants, the seroprevalence rates of HIV, HBV, and syphilis were 15 (5.3\%), 9 (3.2\%), and $5(1.8 \%)$, respectively. Twenty six (9.3\%) of the participants tested positive for any one of the STIs, and $3(1.1 \%)$ of the women had HIV and syphilis coinfections. History of multiple sexual partners (AOR 3.42, 95\% CI: 1.6-11.63) and STIs (AOR 3.7; 95\% CI: 1.70-13.45) were significantly associated with HIV infection. Likewise, history of abortion (AOR 7.65, 95\% CI: 1.17-49.74), tattooing (AOR 9.72, 95\% CI: 1.41-66.73), and rape (AOR 9.72, 95\% CI: 1.41-66.73) were significantly associated with hepatitis B virus infection. Husband history of multiple sexual partners (AOR 20.9, 95\% CI: 1.8-241.8) was significantly associated with syphilis infection. The educational level of participants, history of tattooing (AOR 6.24, 95\% CI: 1.79-21.7), and history of multiple sexual partners (AOR 5.15, 95\% CI: 1.68-15.7) were independent predictors of infection with any one of the STIs. Conclusion. There is still a high burden of HIV, HBV, and syphilis among pregnant mothers in Ethiopia. History of multiple sexual partners, abortion, rape, and tattooing was a significant predictor of STIs.
\end{abstract}

\section{Introduction}

The global burden of HIV, HBV, and syphilis illnesses is a widespread problem among pregnant women, which in turn compromises the quality of life, reproductive health, and infant and child health $[1,2]$.

HIV remains to be the major public health problem of the world. At the end of 2020, there were 37.7 million people living with HIV with 680,000 HIV-related deaths and 1.5 million new infections [3]. Pregnant women account for approximately $48 \%$ of all new HIV infections and approxi- mately half (52\%) of all individuals living with HIV worldwide, and the infection is the leading cause of death among women of reproductive age $[2,4]$. In Ethiopia, at the end of 2017, there were 650,000 people living with HIV with 17,200 AIDS-related deaths and 14,500 new infections [5]. In a meta-analysis, the pooled seroprevalence of HIV among pregnant women in Ethiopia was 5.74\% [6].

HIV has adverse pregnancy outcomes on pregnant women like intrauterine growth restriction (IUGR) (20.5\%), preterm birth (25.0\%), and cesarean delivery $(45.5 \%)[7,8]$. Besides, without any intervention, transmission rates of HIV 
from mother to child range from $15 \%$ to $45 \%$. Similarly, $\mathrm{HIV}$-infected and untreated children are at the highest risk of mortality within the first 3 months of life and up to $50 \%$ die within 2 years [3].

Globally, perinatal HBV spread accounts for an estimated $21 \%$ of HBV-related child deaths [9]. Africa has the biggest number of confirmed carriers after Asia and is considered a region of high endemicity [10]. It is assessed that between 70 and $95 \%$ of the adult population show evidence of past exposure to $\mathrm{HBV}$ infection and the prevalence of $\mathrm{HBV}$ disease among pregnant ladies ranges from 1.6\% to13.8\% [10]. Globally, 1.5 million people were newly infected with HBV in 2019, with 820,000 HBV infectionrelated deaths [3]. In Ethiopia, the pooled prevalence of $\mathrm{HBV}$ among the general population was $7.4 \%[11,12]$, while it was $4.75 \%$ among the pregnant women $[13,14]$ that could be classified as WHO intermediate endemicity.

Syphilis, which is caused by Treponema pallidum, is still a global health problem despite the widely available methods of screenings and treatments. Globally, an estimated $0.8 \%$ of pregnant women and, in the African region, 2.9\% of pregnant women are infected with syphilis [15]. In Ethiopia, the seroprevalence of syphilis among different subgroups of populations ranges from 1 to $10.9 \%$ [16]. The pooled seroprevalence of syphilis among pregnant women in Ethiopia was found to be $2.32 \%$ [17].

Untreated syphilis leads to an increment within the hazard of mother-to-child transmission (MTCT) of HIV. It has unfavorable pregnancy results, such as abortion, intrauterine fetal death, stillbirth, growth restriction, low birth weight, neonatal death, and congenital syphilis, due to untreated STIs $[15,18,19]$. Ethiopia is one of the three sub-Saharan countries with the highest numbers of adverse pregnancy outcomes caused by syphilis [19].

As recommended by the Global Health Sector Strategy on STI 2016-2021, diagnosing and treating these devastating etiologic agents at an early stage may result in preventing the spread of such infections and complications, for pregnant women as well as for their newborn infants [20]. Therefore, this study was designed to investigate the seroprevalence of HIV, HBV, and syphilis infections and their risk factors among pregnant women in Addis Ababa, Ethiopia.

\section{Materials and Methods}

2.1. Study Area, Design Period, and Population. A crosssectional study design was conducted from February 1, 2021, to March 30, 2021, among 281 pregnant women in four randomly selected public hospitals, namely, Tikur Anbessa Specialized Hospital, Zewuditu Memorial Hospital, Ghandi Memorial Hospital, and St. Petros Hospital found in Addis Ababa, the capital city of Ethiopia. The city has 13 public hospitals, of which the four hospitals were selected by the lottery method. The study population was all pregnant women attending antenatal care at public hospitals in Addis Ababa during the study period. Women attending antenatal care who were willing to participate in the study and residing in Addis Ababa for at least 6 months were included in the study.
Pregnant women who have severe health problems during the data collection period were excluded.

2.2. Sample Size Determination and Sampling Procedures. The sample size was determined using a single-population proportion formula by taking $6.5 \%$ the highest prevalence of HIV infection from the recent study in Dessie, Ethiopia [21], with an assumption of a 95\% level of confidence and $3 \%$ margin of error. Accordingly, after adding the $10 \%$ nonresponse rate, a total of 286 pregnant women were included in the study.

The total sample size $(n=286)$ was allocated to each hospital proportionally based on the previous three-month average number of pregnant women attending antenatal care in each hospital. The study participants were chosen using the systematic random sampling method. The $k^{\text {th }}$ interval of systematic sampling was determined by dividing the previous 3-month average number of mothers on antenatal care with the required sample size from each hospital.

2.3. Data Collection and Laboratory Diagnosis. An interviewadministered questioner was used to collect data on the sociodemographic factors, risky sociocultural and behavioral practices, and hospital-related factors. All antenatal pregnant mothers receive routine laboratory diagnostic tests for HIV, $\mathrm{HBV}$, and syphilis. Following the interview, 10 milliliters of venous blood sample was collected by sterile vein puncture procedure from each participant. The blood was centrifuged for $5 \mathrm{~min}$ at 300 revolutions per minute, and the serum sample was used for HIV, HBV, and syphilis screening.

HIV testing was done according to the national algorithm recommended by the Federal Ministry of Health of Ethiopia. Rapid HIV tests: HIV $(1+2)$ rapid test strip Wantai (Beijing Wantai Biological Pharmacy Enterprise Co. Ltd., China) as the screening test, Uni-Gold HIV test ${ }^{\circledR}$ (Trinity Biotech Inc., Wicklow, Ireland) as a confirmatory test for positive samples, and VIKIA ${ }^{\circledR}$ HIV $1 / 2$ (bioMérieux, SA, Marcy L'Etoile, France) as a tie-breaker test, were conducted in a series algorithm.

Sera were screened for syphilis using the rapid plasma reagin (RPR) test (Human GmbH-Wiesbaden, Germany), and the positives were retested using Treponema pallidum haemagglutination assay (TPHA) test (Guangzhou Wondfo Biotech Co. Ltd., Guangzhou, People's Republic of China). Pregnant women who tested positive for both RPR and TPHA were considered having Treponema pallidum infection.

All sera were tested for hepatitis B surface antigen (HBsAg) using rapid diagnostic tests that employ the principle of immunochromatography (SD Bioline, Yongin, Korea), which is believed to have an accuracy of above $98 \%$ and the best test to detect the current infection among pregnant women [22].

2.4. Data Quality Control and Analysis. For the data quality control, the questionnaire was checked and pretested before the actual data collection has been started. All the laboratory tests were performed following the manufacturers' protocol and quality in-built control assays. After checking for completeness and consistency of the collected information, the 
data was cleaned, coded, entered into Epi-Data 3.1, and transferred to Statistical Package for Social Sciences (SPSS) version 25 for analysis. The descriptive statistics (means, percentages, or frequency) was calculated, and the binary logistic regression analysis was used to see the relationship between dependent and independent variables. Variables having a $p$ value of $<0.20$ in bivariate analysis were considered to have an association with the dependent variable and fitted into the multivariate analysis model. The strength of the association was measured using an odds ratio and interpreted by considering the $95 \%$ confidence interval, and $p<0.05$ was a statistically significant risk factor in multivariate analysis.

2.5. Ethical Statement. Ethical approval was obtained from the Institutional Review Board of the School of Nursing and Midwifery, Addis Ababa University. Written consent was secured from study participants after a thorough explanation and understanding of the purpose of the study. Confidentiality and privacy of participants were maintained throughout the study process.

\section{Results}

3.1. Sociodemographic and Some Obstetric Characteristics of Study Participants. The study approached a total of 286 pregnant women, of which 281 participants completed the study, with a $98.2 \%$ response rate. The mean age of the participants was 27.5 where majority (199) (70.8\%) of them were between 19 and 29 years old. Most of the study participants were married $(256,91.1 \%)$, and $116(41.3 \%)$ attend secondary school. Among the study participants, 116 (41.3\%) were government employees, 161 (57.3\%) of them were multiparous, and 120 (42.7\%) of them were multigravidas (Table 1).

3.2. Seroprevalence of $H I V, H B V$, and Syphilis Infections among the Study Participants. HIV, HBV, and syphilis were found to be prevalent in 15 (5.3\%), 9 (3.2\%), and 5 (1.8\%) of the study participants, respectively, out of which $3(1.1 \%)$ had HIV/syphilis coinfection. There were no coinfections with HIV/HBV, HBV/syphilis, or HIV/HBV/syphilis. Among the 281 pregnant women, $26(9.3 \%)$ were found to be infected with any one of the three STIs.

\subsection{Risky Hospital-Related, Sociocultural, and Behavioral} Factors among Pregnant Women. Among the study participants, $29.9 \%$ of them had a history of hospital admission, $29.2 \%$ had history of operation for a surgical procedure, and $19.2 \%$ of them had a history of blood transfusion. More than $38 \%$ had a history of sexually transmitted infections, $65.1 \%$ have a history of premarital sex, majority $(94.7 \%)$ of them had ear piercing, and $35.9 \%$ of them had history of multiple sexual partners (Table 2).

3.4. Seroprevalence and Associated Risk Factors of HIV, HBV, and Syphilis Infections among the Study Participants. To determine the relationship between each independent variable and HIV, HBV, and syphilis serostatus, binary logistic regression analysis was conducted. The independent vari-
TABLE 1: Sociodemographic and some obstetric characteristics of pregnant mothers visiting antenatal clinics at selected hospitals in Addis Ababa, February, $2021(n=281)$.

\begin{tabular}{lccc}
\hline Variables & Categories & Frequency & Percent \\
\hline \multirow{4}{*}{ Age } & $19-29$ & 199 & 70.8 \\
& $30-39$ & 77 & 27.4 \\
Marital status & $40-49$ & 5 & 1.8 \\
& Single & 25 & 8.9 \\
\multirow{4}{*}{ Educational status } & Married & 256 & 91.1 \\
& No formal education & 34 & 12.1 \\
& Primary school & 55 & 19.6 \\
& Secondary school & 116 & 41.3 \\
Occupation & College and above & 76 & 27 \\
& Employed & 116 & 41.3 \\
& Unemployed & 102 & 36.3 \\
Gravidity & Farmer & 5 & 1.8 \\
& Private & 58 & 20.6 \\
Parity & Primigravida & 24 & 8.5 \\
& Multigravida & 257 & 91.5 \\
& Primiparous & 121 & 43.1 \\
& Multiparous & 160 & 56.9 \\
\hline
\end{tabular}

ables with a $p$ value of $\leq 0.20$ were fitted into the multivariate logistic regression model to identify independent predictors of HIV, HBV, and syphilis infection (Tables 3-5).

3.5. Seroprevalence of HIV and Associated Factors. The total prevalence of HIV was 5.3\% (95\% CI: 2.8-8.2). In bivariate logistic regression, women's history of multiple sexual partners, history of husband multiple sexual partners, history of women STIs, husband history of STIs, and history of surgical operation were correlated with the seroprevalence of HIV infection $(p \leq 0.05)$. However, in multivariate analysis, history of women's STIs and history of husband sexual partner remained independent predictors of HIV infection. Women having a history of STIs were 3.79 times (AOR 3.79; 95\% CI: 1.70-13.45) more likely to be infected by HIV than those who did not have STIs. Likewise, pregnant women whose husbands had a history of multiple sexual partners had more than threefold (AOR 3.42; 95\% CI: 1.60-11.63) chance of acquiring HIV infection compared to their counterparts (Table 3).

3.6. Seroprevalence of Hepatitis B Virus (HBV) and Associated Factors among the Study Participants. The seroprevalence of HBV was 3.2\% (95\% CI: 1.1-5.3). In bivariate logistic regression, history of abortion, dental procedure, hospital admission, and tattooing were associated with seroprevalence of HBV infection $(p \leq 0.05)$. However, in multivariate analysis, history of tattooing, abortion, and rape were found to be independent predictors of HBV infection. Women with a history of tattooing were twelve times (AOR 11.88, 95\% CI: 2.17-16.02) more likely to be infected with HBV compared to those who did not have tattooing. Similarly, having histories of abortion (AOR 7.65, 95\% CI: $1.17-9.74$ ) and rape (AOR 9.72, 95\% CI: 1.41-16.73) was 
TABLE 2: Risky characteristics and distribution of the three STIs among pregnant women attending antenatal care at selected hospitals in Addis Ababa, February to March, $2021(n=281)$.

\begin{tabular}{|c|c|c|c|c|c|c|}
\hline Variables & Categories & Frequency, $n(\%)$ & HIV, $n(\%)$ & HBV, $n(\%)$ & Syphilis, $n(\%)$ & Any STI, $n(\%)$ \\
\hline \multirow{2}{*}{ History of hospital admission } & No & $197(70.1)$ & $9(3.2)$ & $3(1.0)$ & $4(1.4)$ & $14(5.0)$ \\
\hline & Yes & $84(29.9)$ & $6(2.1)$ & $6(2.1)$ & $1(0.4)$ & $12(4.8)$ \\
\hline \multirow{2}{*}{ History of dental procedure } & No & $220(78.3)$ & $9(3.2)$ & $3(1.0)$ & $3(1.0)$ & $14(5.0)$ \\
\hline & Yes & $61(21.7)$ & $6(2.1)$ & $6(2.1)$ & $2(0.7)$ & $12(4.8)$ \\
\hline \multirow{2}{*}{$\begin{array}{l}\text { History of operation for a } \\
\text { surgical problem }\end{array}$} & No & $197(70.1)$ & $7(2.5)$ & $9(3.2)$ & $3(1.0)$ & $18(6.4)$ \\
\hline & Yes & $84(29.9)$ & $8(2.8)$ & $0(0.0)$ & $2(0.7)$ & $8(2.8)$ \\
\hline \multirow{2}{*}{ History of blood transfusion } & No & $227(80.8)$ & $10(3.6)$ & $5(1.8)$ & $3(1.0)$ & $17(6.0)$ \\
\hline & Yes & $54(19.2)$ & $5(1.8)$ & $4(1.4)$ & $2(0.7)$ & $9(3.2)$ \\
\hline \multirow{2}{*}{ History of women STIs } & No & $175(62.3)$ & $4(1.4)$ & $6(2.1)$ & $2(0.7)$ & $12(4.8)$ \\
\hline & Yes & $108(38.4)$ & $11(3.9)$ & $5(1.8)$ & $3(1.0)$ & $14(5.0)$ \\
\hline \multirow{2}{*}{ History of partner STIs } & No & $187(66.5)$ & $6(2.1)$ & $7(2.5)$ & $1(0.4)$ & $13(4.6)$ \\
\hline & Yes & $94(33.5)$ & $9(3.2)$ & $2(0.7)$ & $4(1.4)$ & $13(4.6)$ \\
\hline \multirow{2}{*}{ History of abortion } & No & $235(83.6)$ & $12(4.3)$ & $3(1.0)$ & $4(1.4)$ & $17(6.0)$ \\
\hline & Yes & $46(16.4)$ & $3(1.0)$ & $6(2.1)$ & $1(0.4)$ & $9(3.2)$ \\
\hline \multirow{2}{*}{ History of tattooing } & No & $244(86.8)$ & $12(4.3)$ & $3(1.0)$ & $4(1.4)$ & $16(5.7)$ \\
\hline & Yes & $37(13.2)$ & $3(1.0)$ & $6(2.1)$ & $1(0.4)$ & $10(3.6)$ \\
\hline \multirow{2}{*}{ History of ear piercing } & No & $15(5.3)$ & $1(0.4)$ & $1(0.4)$ & $0(0.0)$ & $2(0.7)$ \\
\hline & Yes & $266(94.7)$ & $14(5.0)$ & $8(2.8)$ & $5(1.8)$ & $24(8.5)$ \\
\hline \multirow{2}{*}{ History of nose piercing } & No & $267(95)$ & $14(5.0)$ & $9(3.2)$ & $5(1.8)$ & $25(8.9)$ \\
\hline & Yes & $14(5.0)$ & $1(0.4)$ & $0(0.0)$ & $0(0.0)$ & $1(0.4)$ \\
\hline \multirow{2}{*}{ History of premarital sex } & No & $98(34.9)$ & $7(2.5)$ & $3(1.0)$ & $5(1.8)$ & $12(4.8)$ \\
\hline & Yes & $183(65.1)$ & $8(2.8)$ & $6(2.1)$ & $0(0.0)$ & $14(5.0)$ \\
\hline \multirow{2}{*}{ Share sharp instruments with others } & No & $172(61.2)$ & $9(3.2)$ & $5(1.8)$ & $0(0.0)$ & $14(5.0)$ \\
\hline & Yes & $109(38.8)$ & $6(2.1)$ & $4(1.4)$ & $5(1.8)$ & $12(4.8)$ \\
\hline \multirow{2}{*}{ History of rape } & No & $212(75.4)$ & $11(3.9)$ & $5(1.8)$ & $2(0.7)$ & $17(6.0)$ \\
\hline & Yes & 69 (24.6) & $4(1.4)$ & $4(1.4)$ & $3(1.0)$ & $9(3.2)$ \\
\hline \multirow{2}{*}{$\begin{array}{l}\text { History of women with multiple } \\
\text { sexual partners }\end{array}$} & No & $180(64.1)$ & $5(1.8)$ & $6(2.1)$ & $1(0.4)$ & $11(3.9)$ \\
\hline & Yes & $101(35.9)$ & $10(3.6)$ & $3(1.0)$ & $4(1.4)$ & $15(5.3)$ \\
\hline \multirow{2}{*}{$\begin{array}{l}\text { History of husband with multiple } \\
\text { sexual partners }\end{array}$} & No & $220(78.3)$ & $8(2.8)$ & $7(2.5)$ & $1(0.4)$ & $16(5.7)$ \\
\hline & Yes & $61(21.7)$ & $7(2.5)$ & $2(0.7)$ & $4(1.4)$ & $10(3.6)$ \\
\hline
\end{tabular}

more than 7 and 9 times more likely to be infected by HBV infection than those who did not have these histories (Table 4).

3.7. Seroprevalence of Syphilis and Associated Factors among the Study Participants. The overall prevalence of syphilis was $1.8 \%$ (95\% CI: 0.4-3.8). In multivariate analysis, women whose partners have history of multiple sexual partners were 20 times (AOR 20, 95\% CI: 1.8-41.8) more likely to be infected by syphilis than their counterparts.

3.8. Seroprevalence and Associated Factors for Any of the 3 STIs (HIV, HBV, and Syphilis). Out of the 281 pregnant women, 26 was found to be reactive for any of the 3 STIs with an overall prevalence of 9.3\% (95\% CI: 6.0-12.8). In bivariate analysis, the educational level of respondents, history of dental procedure, history of blood transfusion, history of abortion, history of tattooing, history of multiple sexual partners, and history of husband's sexual partner were associated with the overall prevalence of STIs. However, in multivariate analysis, the educational level of respondents, history of tattooing, and history of multiple sexual partner were significantly associated with the overall occurrence of sexually transmitted infections. Accordingly, women with an educational level of secondary school were 89\% times (AOR 0.11, 95\% CI: 0.03-0.41) less likely to be infected with STIs than those who did not have formal education. Similarly, pregnant women who have an educational level of college and above were $81 \%$ (AOR 0.19, 95\% CI: 0.04-0.77) less likely to be infected by STIs. Likewise, women having a history of tattooing were 6 times (AOR 6.24; 95\% CI: 1.79-21.70) more likely to be infected by STIs than their counterparts. Furthermore, women with a history of multiple sexual partners had a fivefold chance of getting any one of the STIs than their counterparts (AOR 5.15, 95\% CI:1.68-15.7) (Table 5). 
TABLE 3: Associated factors of HIV infection among pregnant women attending antenatal care at selected public hospitals in Addis Ababa, February to March, $2021(n=281)$.

\begin{tabular}{|c|c|c|c|c|c|c|c|}
\hline \multirow{2}{*}{ Variables } & \multirow{2}{*}{ Category } & \multirow{2}{*}{ Frequency } & \multicolumn{2}{|c|}{ HIV serostatus } & \multirow{2}{*}{$\begin{array}{c}\text { Bivariate analysis } \\
\text { COR }(95 \% \mathrm{CI})\end{array}$} & \multicolumn{2}{|c|}{ Multivariate analysis } \\
\hline & & & No, $n(\%)$ & Yes, $n(\%)$ & & AOR (95\% CI) & $p$ value \\
\hline \multirow{2}{*}{ Marital status } & Single & 25 & $22(7.8)$ & $3(1.0)$ & 1 & 1 & - \\
\hline & Married & 256 & $244(87)$ & $12(4.0)$ & $0.361(0.095-1.37)$ & $0.56(0.10-3.18)$ & 0.51 \\
\hline \multirow{2}{*}{ Dental procedure } & No & 220 & $211(75)$ & $9(3.2)$ & 1 & 1 & - \\
\hline & Yes & 61 & $55(19.5)$ & $6(2.1)$ & $2.55(0.873-7.492)$ & $1.53(0.42-5.52)$ & 0.51 \\
\hline \multirow{2}{*}{ Hx of surgical procedure } & No & 197 & $190(68)$ & $7(2.4)$ & 1 & 1 & \\
\hline & Yes & 84 & $76(27)$ & $8(2.8)$ & $2.85(1.1-8.154)$ & $2.83(0.88-9.10)$ & 0.08 \\
\hline \multirow{2}{*}{ Hx of blood transfusion } & No & 227 & $217(77)$ & $10(4.0)$ & 1 & 1 & - \\
\hline & Yes & 54 & 49 (17.4) & $5(1.7)$ & $2.21(0.72-6.76)$ & $2.11(0.54-8.25)$ & 0.28 \\
\hline \multirow{2}{*}{ History of woman STIs } & No & 173 & $169(60)$ & $4(1.4)$ & 1 & 1 & - \\
\hline & Yes & 108 & $97(34.5)$ & $11(4)$ & $4.79(1.48-15.45)^{*}$ & $3.7(1.70-13.45)$ & $0.03^{* *}$ \\
\hline \multirow{2}{*}{ History of husband STIs } & No & 187 & $181(64)$ & $6(2.1)$ & 1 & 1 & - \\
\hline & Yes & 94 & $85(30.2)$ & $9(3.2)$ & $3.19(1.10-9.26)^{*}$ & $2.07(0.63-6.71)$ & 0.22 \\
\hline \multirow{2}{*}{ Hx of husband with multiple sexual partners } & No & 220 & $212(75)$ & $8(2.8)$ & 1 & 1 & - \\
\hline & Yes & 61 & $54(19.2)$ & $7(2.4)$ & $3.43(1.19-9.89)^{*}$ & $3.42(1.6-11.63)$ & $0.04^{* *}$ \\
\hline \multirow{2}{*}{ Hx of woman with multiple sexual partners } & No & 180 & $175(62)$ & $5(1.7)$ & 1 & 1 & - \\
\hline & Yes & 101 & $91(32.3)$ & $10(4.0)$ & $3.84(1.27-11.58)^{*}$ & $2.20(0.66-7.31)$ & 0.19 \\
\hline
\end{tabular}

${ }^{*}$ In a bivariate analysis, $p \leq 0.05$ is statistically significant. ${ }^{* *}$ In multivariate analysis, $p \leq 0.05$ is statistically significant; 1 : reference category; STIs: sexually transmitted infections; Hx: history.

TABLE 4: Associated factors of hepatitis B infection among pregnant women attending antenatal care at selected public hospitals in Addis Ababa, February to March, $2021(n=281)$.

\begin{tabular}{|c|c|c|c|c|c|c|c|}
\hline \multirow{2}{*}{ Variables } & \multirow{2}{*}{ Category } & \multirow{2}{*}{ Frequency } & \multicolumn{2}{|c|}{ HBV serostatus } & \multirow{2}{*}{$\begin{array}{c}\text { Bivariate analysis } \\
\text { COR }(95 \% \text { CI })\end{array}$} & \multicolumn{2}{|c|}{ Multivariate analysis } \\
\hline & & & No, $n(\%)$ & Yes, $n(\%)$ & & AOR $(95 \% \mathrm{CI})$ & $p$ value \\
\hline \multirow{2}{*}{ History of dental procedure } & No & 220 & $217(98.6)$ & $3(1.4)$ & 1 & 1 & - \\
\hline & Yes & 61 & $55(90.2)$ & $6(9.8)$ & $7.89(1.9-32.55)^{*}$ & $4.86(0.79-29.63)$ & 0.08 \\
\hline \multirow{2}{*}{ History of blood transfusion } & No & 227 & $222(97.8)$ & $5(2.2)$ & 1 & 1 & - \\
\hline & Yes & 54 & $50(92.6)$ & $4(7.4)$ & $3.55(0.92-13.7)^{*}$ & $1.98(0.32-12.10)$ & 0.45 \\
\hline \multirow{2}{*}{ History of hospital admission } & No & 197 & $194(98.5)$ & $3(1.5)$ & 1 & 1 & - \\
\hline & Yes & 84 & $78(92.9)$ & $6(7.1)$ & $4.97(1.2-20.38)^{*}$ & $1.90(0.34-10.51)$ & 0.46 \\
\hline \multirow{2}{*}{ History of abortion } & No & 235 & $232(98.7)$ & $3(1.3)$ & 1 & 1 & - \\
\hline & Yes & 46 & $40(87.0)$ & $6(13.0)$ & $11.6(2.7-48.27)^{*}$ & $7.65(1.17-19.74)$ & $0.03^{* *}$ \\
\hline \multirow{2}{*}{ History of rape } & No & 212 & $207(97.6)$ & $5(2.4)$ & 1 & 1 & - \\
\hline & Yes & 69 & $65(94.2)$ & $4(5.8)$ & $2.54(0.66-9.76)$ & $9.72(1.41-16.73)$ & $0.02^{* *}$ \\
\hline \multirow{2}{*}{ History of tattooing } & No & 244 & $241(98.8)$ & $3(1.2)$ & 1 & 1 & - \\
\hline & Yes & 37 & $31(83.8)$ & $6(16.2)$ & $15.54(3.7-65.3)^{*}$ & $11.8(2.17-16.02)$ & $0.004^{* *}$ \\
\hline
\end{tabular}

${ }^{*}$ In a bivariate analysis, $p \leq 0.05$ is statistically significant. ${ }^{* *}$ In multivariate analysis, $p \leq 0.05$ is statistically significant; 1 : reference category.

\section{Discussion}

This study pursued to estimate the prevalence of the 3 STIs and identify the risk factors of infection of HIV, HBV, and syphilis among pregnant women attending antenatal care at public hospitals in Addis Ababa, Ethiopia.

In the present study, the seroprevalence of HIV among pregnant women was $5.3 \%$, which is in line with studies done in Cameroon (5.0\%) [23], sub-Saharan African coun- tries (5.3\%) [24], and Ethiopia (5.74\%) [6]. This finding was lower than the previous studies done in China (6.6\%) [25], Cameroon (13.1\%) [26], Gondar (11.2\%) [27], and Dessie (6.5\%) [21]. However, it is higher than a study done in Addis Ababa (4.8\%) [6], Gondar (4.1\%) [28], Sudan (1.13\%) [29], Guatemala (0.21\%) [30], and Angola (2.6\%) [31]. The variations might be the reflections of differences in sociocultural practices, accessibility to the health care system, risky sexual and behavioral practices, and awareness of 
TABLE 5: Associated factors of any one of the STIs among pregnant women attending antenatal care at selected public hospitals in Addis Ababa, February to March, $2021(n=281)$.

\begin{tabular}{|c|c|c|c|c|c|c|c|}
\hline \multirow{2}{*}{ Variables } & \multirow{2}{*}{ Category } & \multirow{2}{*}{ Frequency } & \multicolumn{2}{|c|}{ Any STI serostatus } & \multirow{2}{*}{$\begin{array}{c}\text { Bivariate analysis } \\
\text { COR }(95 \% \mathrm{CI})\end{array}$} & \multicolumn{2}{|c|}{ Multivariate analysis } \\
\hline & & & No, $n(\%)$ & Yes, $n(\%)$ & & $\operatorname{AOR}(95 \% \mathrm{CI})$ & $p$ value \\
\hline \multirow{2}{*}{ Gravidity } & Primigravida & 24 & $21(87.5)$ & $3(12.5)$ & 1 & 1 & - \\
\hline & Multigravida & 257 & $234(91.1)$ & $23(8.9)$ & $0.69(0.19-2.48)$ & & \\
\hline \multirow{2}{*}{ Parity } & Primiparous & 121 & $106(87.6)$ & $15(12.4)$ & 1 & 1 & - \\
\hline & Multiparous & 160 & $149(93.1)$ & $11(6.9)$ & $0.52(0.23-1.18)$ & $0.55(0.17-1.76)$ & 0.32 \\
\hline \multirow{4}{*}{ Educational level } & No formal education & 34 & $22(64.7)$ & $12(35.3)$ & 1 & 1 & - \\
\hline & Primary school & 55 & $55(100)$ & $0(0.0)$ & & & \\
\hline & Secondary school & 116 & $109(94)$ & $7(6)$ & $0.11(0.04-0.33)^{*}$ & $0.11(0.03-0.41)$ & $0.004^{* *}$ \\
\hline & College and above & 76 & $69(90.8)$ & $7(9.2)$ & $0.18(0.05-0.53)^{*}$ & $0.19(0.04-0.77)$ & $0.020^{* *}$ \\
\hline \multirow{2}{*}{ Hx of hospital admission } & No & 197 & $183(92.9)$ & $14(7.1)$ & 1 & 1 & - \\
\hline & Yes & 84 & $72(85.7)$ & $12(14.3)$ & $2.18(0.96-4.93)$ & $1.59(0.50-5.11)$ & 0.43 \\
\hline \multirow{2}{*}{ History of dental procedure } & No & 220 & $206(93.6)$ & $14(6.4)$ & 1 & 1 & - \\
\hline & Yes & 61 & $49(80.3)$ & $12(19.7)$ & $3.60(1.57-8.28)^{*}$ & $2.31(0.70-7.54)$ & 0.16 \\
\hline \multirow{2}{*}{ History of blood transfusion } & No & 227 & $210(92.5)$ & $17(7.5)$ & 1 & 1 & - \\
\hline & Yes & 54 & $45(83.3)$ & $9(16.7)$ & $2.47(1.04-5.90)^{*}$ & $2.76(0.80-9.55)$ & 0.11 \\
\hline \multirow{2}{*}{ History of woman STIs } & No & 173 & $161(93.1)$ & $12(6.9)$ & 1 & 1 & - \\
\hline & Yes & 108 & $94(87)$ & $14(7)$ & $2.0(0.89-4.50)$ & $1.67(0.54-5.11)$ & 0.37 \\
\hline \multirow{2}{*}{ History of husband STIs } & No & 187 & $174(93)$ & $13(7)$ & 1 & 1 & - \\
\hline & Yes & 94 & $81(86.2)$ & $13(13.8)$ & $2.15(0.95-4.84)$ & $2.86(0.97-8.40)$ & 0.06 \\
\hline \multirow{2}{*}{ History of abortion } & No & 235 & $218(92.8)$ & $17(7.2)$ & 1 & 1 & - \\
\hline & Yes & 46 & $37(80.4)$ & $9(19.6)$ & $3.11(1.30-7.52)^{*}$ & $0.75(0.16-3.39)$ & 0.71 \\
\hline \multirow{2}{*}{ History of tattooing } & No & 244 & $228(93.4)$ & $16(6.6)$ & 1 & 1 & - \\
\hline & Yes & 37 & $27(73)$ & $10(27)$ & $5.28(2.18-12.79)^{* * *}$ & $6.24(1.79-21.7)$ & $0.004^{* *}$ \\
\hline \multirow{2}{*}{ History of premarital sex } & No & 98 & $86(87.8)$ & $12(12.2)$ & 1 & 1 & - \\
\hline & Yes & 183 & $169(92.3)$ & $14(7.7)$ & $0.59(0.26-1.34)$ & $0.34(0.10-1.16)$ & 0.08 \\
\hline \multirow{2}{*}{$\begin{array}{l}\text { Hx of multiple } \\
\text { sexual partners }\end{array}$} & No & 180 & $169(93.9)$ & $11(6.1)$ & 1 & 1 & - \\
\hline & Yes & 101 & $86(85.1)$ & $15(14.9)$ & $2.68(1.18-6.08)^{*}$ & $5.15(1.68-15.7)$ & $0.004^{* *}$ \\
\hline \multirow{2}{*}{ History of rape } & No & 212 & $195(92)$ & $17(8)$ & 1 & 1 & - \\
\hline & Yes & 69 & $60(87)$ & $9(13)$ & $1.72(0.72-4.06)$ & $2.69(0.80-8.98)$ & 0.11 \\
\hline \multirow{2}{*}{$\begin{array}{l}\text { Hx of husband with } \\
\text { multiple sexual partners }\end{array}$} & No & 220 & $204(92.7)$ & $16(7.3)$ & 1 & 1 & - \\
\hline & Yes & 61 & $51(83.3)$ & $10(16.4)$ & $2.50(1.07-5.83)^{*}$ & $1.92(0.59-6.26)$ & 0.28 \\
\hline
\end{tabular}

${ }^{*}$ In a bivariate analysis, $p \leq 0.05$ is statistically significant. ${ }^{* *}$ In multivariate analysis, $p \leq 0.05$ is statistically significant; 1 : reference category. Sexual history of the women's husband.

HIV infection. This study indicates that HIV is still an important public health concern among pregnant women in Addis Ababa and suggests that there is a need to strengthen intervention efforts including health education towards voluntary counseling and testing, modes of transmission, prevention, and mother-to-child transmission of HIV infection. The present study demonstrated that study participants with a history of other STIs were 3.79 times more likely to be infected with HIV than those without previous history of STIs. This finding was supported by a research done in Dessie, Ethiopia [21], and Addis Ababa [6] in which the history of STIs was associated with HIV infection. This might be because STIs create ulcer on the skin and mucous membrane which in turn facilitates the acquisition of HIV infection. Pregnant women having a husband with a history of multiple sexual partners have 3.42 times more chance to acquire HIV infection compared to their counterparts. Studies conducted in Dessie, Ethiopia [21], Addis Ababa [6], Uganda [32], Cameroon [26], and Southwest Nigeria [33] showed that the habit of multiple sexual practices was significantly associated with the acquisition of HIV infection. This may be because husbands of pregnant women have risky sexual behavior that facilitates the acquisition of HIV both for them and for the women.

In this study, the overall prevalence of hepatitis B infection was $3.2 \%$, which can be graded as intermediate endemicity according to WHO criteria [11]. This result is consistent with a study conducted in Kenya (3.8\%) [34], Tanzania (3.9\%) [35], Dawro (3.5\%) [36], and Bahir Dar, Ethiopia (3.8\%) [37]. Conversely, this finding was lower than studies done in Kano, Nigeria (7.9\%) [33], Ghana (9.5\%) [38], Angola (7.5\%) [31], Solomon Islands (13.8\%) [39], 
Deder (6.9\%) [40], Arbaminch (4.3\%) [41], Dessie (4.7\%), Yirgalem (7.2\%) [42], and southern Ethiopia (7.8\%) [43]. However, it is higher than studies reported in Egypt (1.6\%) [44] and Sudan (2.93\%) [29]. The differences observed in the prevalence of hepatitis B virus prevalence across a different country might be due to differences in sociodemographic characteristics, risky sexual and behavioral practices, sociocultural environment, and traditional operations where the study participants are residing.

According to the current study, women who had history of abortion were 7.65 times more likely to be infected with $\mathrm{HBV}$ than women who had never had an abortion. Similarly, research conducted in Nigeria [33] and Deder [40] showed that women history of abortion was substantially linked with HBV infection. Participants in the study who had a history of rape were found to be 9.72 times more infected than their peers. The present study showed that pregnant women with a history of tattooing were almost twelve times more infected than those who did not have this phenomenon. This could be because tattooing was done in a conventional fashion, with the risk of using common sharp tools, which would facilitate the spread of hepatitis B virus infection.

The total frequency of syphilis among pregnant women was 1.8 percent in this study. This finding was consistent with research done in Bahir Dar (2.9\%) [45]. Yet, the prevalence of syphilis is higher than $0.6 \%$ of Dessie, Ethiopia [21]. Compared to other countries, the observed $1.8 \%$ seroprevalence of syphilis is lower than the $5.7 \%$ prevalence in Cameroon [23], 2.9\% in sub-Saharan African countries [15], 3.2\% in the east African region [15], and 3.8\% in South African regions [15]. The lower prevalence of syphilis among pregnant women might be attributed to variations in sexual and behavioral practices, difference in access to treatment of STIs, the difference in cultural practice, and the increase in syphilis screening at every antenatal care visit.

The present study showed that pregnant women whose husbands have a history of multiple sexual partners were significant risk factors for the acquisition of syphilis infection. Women whose husbands/partners have a history of having a new sex partner in the last 6 months were 20.97 times more infected with syphilis infection. Similarly, studies in the Ekiti state, Nigeria [46], and Bahir Dar [45] showed that a history of multiple sexual partners of women had an association with syphilis infection.

In this study, pregnant women having an educational level of secondary and above the secondary were less likely to be infected with sexually transmitted infections than those who did not have formal education. This may be because respondents might have an awareness regarding sexually transmitted infections, mother-to-child transmission, prevention, and complications of STIs in different youth and schools' anti-AIDS and reproductive health clubs.

\section{Conclusion and Recommendation}

In this study, the history of multiple sexual partners, history of tattooing, history of abortion, history of sexually transmitted infections, and history of rape were significantly associated with HIV, hepatitis B virus, and syphilis infection.
There is still a high burden of HIV, HBV, and syphilis among antenatal mothers in Ethiopia. Therefore, it is important to strengthen the existing antenatal care services and screen STIs among all pregnant women at every antenatal care visit.

\author{
Abbreviations \\ ANC: Antenatal care \\ AOR: Adjusted odds ratio \\ CI: $\quad$ Confidence interval \\ EDHS: Ethiopia demographic and health survey \\ HbsAg: Hepatitis B surface antigen \\ HBV: Hepatitis B virus \\ HIV: Human immunodeficiency virus \\ IRB: Institutional review board \\ MTCT: Mother-to-child transmission \\ OR: Odds ratio \\ SPSS: Statistical package social sciences \\ STIs: Sexually transmitted infections \\ WHO: World Health Organization.
}

\section{Data Availability}

The data used to support the findings of this study are available from the corresponding author upon request. The source of this article is a thesis found in Addis Ababa University Institutional repository at http://etd.aau.edu.et/ handle/123456789/28585 [47].

\section{Disclosure}

The research did not receive specific funding but was performed as part of the employment of the authors. Ababa University and Dilla University were not involved in the manuscript writing, editing, approval, or decision to publish, and they do not have any conflicts of interest in this work.

\section{Conflicts of Interest}

The authors declare no conflict of interests for this work. Mr. Kassa Genetu was a graduate student in Addis Ababa University, and his salary was covered by Dilla University. Mr. Kerebih Abere and Dr. Erdaw Tachbele are employees of Addis Ababa University and were academic advisors of the graduate student and received their salary from the University.

\section{Authors' Contributions}

All authors made equal contributions to the conception, design, execution, acquisition of data, analysis, and interpretation; took part in drafting, revising, or critically reviewing the article; gave final approval of the version to be published on the present journal; and agree to be accountable for all aspects of the work. 


\section{Acknowledgments}

We are thankful to Addis Ababa University for the financial support. We are indebted to the staff of Tikur Anbessa Specialized Hospital, Zewuditu Memorial Hospital, Ghandi Memorial Hospital, and St. Petros Hospitals for their valuable contribution. Finally, the authors are also thankful to the supervisors, data collectors, and study participants. We would also like to acknowledge Addis Ababa University institutional repository that stores the thesis and allows retrieving this article from the thesis.

\section{References}

[1] F. Geo, K. C. C. Brooks, J. S. Butel, and A. Stephen, Jawetz, Melnick Adelberg's Medical Microbiology, S. Riedel, S. Morse, T. Mietzner, and S. Miller, Eds., McGraw-Hill, United States, 28 edn edition, 2019.

[2] M. Taylor, M. Alonso-González, B. Gómez, E. Korenromp, and N. Broutet, "World Health Organization global health sector strategy on sexually transmitted infections: an evidence-toaction summary for Colombia," Revista Colombiana de Obstetricia y Ginecología, vol. 68, no. 3, pp. 193-201, 2017.

[3] World Health Organization, "Global progress report on HIV, viral hepatitis and sexually transmitted infections, 2021," in Accountability for the global health sector strategies 20162021: actions for impact, World Health Organization, Geneva, 2021, Licence: CC BY-NC-SA 3.0 IG.

[4] J. Rowley, S. Vander Hoorn, E. Korenromp et al., "Chlamydia, gonorrhoea, trichomoniasis and syphilis: global prevalence and incidence estimates, 2016," Bulletin of the World Health Organization, vol. 97, no. 8, pp. 548-562P, 2019.

[5] GBD HIV collaborators, "Global, regional, and national incidence, prevalence, and mortality of HIV, 1980-2017, and forecasts to 2030, for 195 countries and territories: a systematic analysis for the global burden of diseases, injuries, and risk factors study 2017," Lancet HIV, vol. 6, no. 12, pp. e831-e859, 2019.

[6] D. Geremew, F. Tajebe, S. Ambachew, A. Endalamaw, and S. Eshetie, "Seroprevalence of HIV among pregnant women in Ethiopia: a systematic review and meta-analysis," $B M C$ Research Notes, vol. 11, no. 1, 2018.

[7] Central Statistical Agency (CSA) [Ethiopia] and ICF, Ethiopia Demographic and Health Survey, CSA and ICF, Addis Ababa, Ethiopia, and RockvilleMaryland, USA, 2016.

[8] O. Joseph, O. Biodun, and E. Michael, "Pregnancy outcome among HIV positive women receiving antenatal HAART versus untreated maternal HIV infection," Journal of the College of Physicians and Surgeons-Pakistan, vol. 21, no. 6, pp. 356359, 2011.

[9] P. Bayo, E. Ochola, C. Oleo, and A. D. Mwaka, "High prevalence of hepatitis B virus infection among pregnant women attending antenatal care: a cross-sectional study in two hospitals in northern Uganda," BMJ Open, vol. 4, no. 11, 2014.

[10] C. Katamba, T. Chungu, and C. Lusale, "HIV, syphilis and hepatitis B coinfections in Mkushi, Zambia: a cross-sectional study," F1000Research, vol. 8, no. 562, 2019.

[11] L. Breakwell, C. Tevi-Benissan, L. Childs, R. Mihigo, and R. Tohme, "The status of hepatitis B control in the African region," The Pan African Medical Journal, vol. 27, 2017.
[12] Y. Belyhun, M. Maier, A. Mulu, E. Diro, and U. G. Liebert, "Hepatitis viruses in Ethiopia: a systematic review and metaanalysis," BMC Infectious Diseases, vol. 16, no. 1, p. 761, 2016.

[13] K. Kebede, D. Abateneh, and A. Belay, "Hepatitis B virus infection among pregnant women in Ethiopia: a systematic review and meta-analysis of prevalence studies," BMC Infectious Diseases, vol. 18, no. 1, 2018.

[14] A. Alemu, L. Zeleke, B. Aynalem, and G. Kassa, "Hepatitis B virus infection and its determinants among pregnant women in Ethiopia: a systematic review and meta-analysis," Infectious Diseases in Obstetrics and Gynecology, vol. 2020, Article ID 9418475, 11 pages, 2020.

[15] S. Hussen and B. T. Tadesse, "Prevalence of syphilis among pregnant women in sub-Saharan Africa: a systematic review and meta-analysis," BioMed Research International, vol. 2019, Article ID 4562385, 10 pages, 2019.

[16] B. Eticha, Z. Sisay, A. Alemayehu, and T. Shimelis, "Seroprevalence of syphilis among HIV-infected individuals in Addis Ababa, Ethiopia: a hospital-based cross-sectional study," BMJ Open, vol. 3, no. 4, 2013.

[17] H. Geremew and D. Geremew, "Sero-prevalence of syphilis and associated factors among pregnant women in Ethiopia: a systematic review and meta-analysis," Systematic Reviews, vol. 10, no. 1, p. 223, 2021.

[18] World Health Organization, Report on global sexually transmitted infection surveillance, World Health Organization, Geneva, 2018, Licence: CC BY-NC-SA 3.0 IGO.

[19] A. Kuznik, A. G. Habib, Y. C. Manabe, and M. Lamorde, "Estimating the public health burden associated with adverse pregnancy outcomes resulting from syphilis infection across 43 countries in sub-Saharan Africa," Sexually Transmitted Diseases, vol. 42, no. 7, pp. 369-375, 2015.

[20] World Health Organization, "Global health sector strategy on sexually transmitted infections 2016-2021," August 2021, http://www.who.int/reproductivehealth/publications/rtis/ ghss-stis/en/.

[21] H. Nuru, N. Nurahmed, Y. D. Teka, F. Mohammed, G. Ferede, and W. Abebe, "Seroprevalence and associated risk factors of human immunodeficiency virus, hepatitis B virus and syphilis among pregnant women attending Dessie referral hospital, Northeast Ethiopia," International Journal of Scientific Reports, vol. 6, no. 11, 2020.

[22] J. Jin, "Screening for hepatitis B in pregnant women," JAMA, vol. 322, no. 4, 2019.

[23] C. B. Sama, V. F. Feteh, M. Tindong, J. T. Tanyi, N. M. Bihle, and F. F. Angwafo 3rd, "Prevalence of maternal HIV infection and knowledge on mother-to-child transmission of HIV and its prevention among antenatal care attendees in a rural area in northwest Cameroon," PLoS One, vol. 12, no. 2, 2017.

[24] J. W. Eaton, T. M. Rehle, S. Jooste et al., "Recent HIV prevalence trends among pregnant women and all women in sub-Saharan Africa," AIDS, vol. 28, Supplement 4, pp. S507S514, 2014.

[25] S. Yang, C. Yang, Q. Liao et al., “Analysis of HIV prevalence among pregnant women in Liangshan prefecture, China, from 2009 to 2015," PLoS One, vol. 12, no. 9, 2017.

[26] J. H. Fouedjio, F. Y. Fouelifack, L. D. Fouelifa, and R. E. Mbu, "Prevalence and associated factors of HIV infection among pregnant women attending antenatal care at the Yaoundé central hospital," International Journal of Reproduction, Contraception, Obstetrics and Gynecology, vol. 6, no. 7, p. 2698, 2017. 
[27] M. Endris, T. Deressa, Y. Belyhun, and F. Moges, "Seroprevalence of syphilis and human immunodeficiency virus infections among pregnant women who attend the University of Gondar teaching hospital, Northwest Ethiopia: a cross sectional study," BMC Infectious Diseases, vol. 15, no. 1, p. 111, 2015.

[28] B. Biadgo, A. Hassen, M. Getaneh et al., "Syphilis and human immunodeficiency virus infections among pregnant women attending antenatal care clinic of Gondar family guidance association, Northwest Ethiopia: implication for prevention of mother to child transmission," Reproductive Health, vol. 16, no. 1, p. 27, 2019.

[29] S. M. Elkheir, Z. O. E. Babiker, S. K. Elamin et al., "Seroprevalence of maternal HIV, hepatitis B, and syphilis in a major maternity hospital in North Kordofan, Sudan," International journal of STD \& AIDS, vol. 29, no. 13, pp. 1330-1336, 2018.

[30] B. Samayoa, M. R. Anderson, K. P. Alonso Pacheco et al., "Seroprevalence of HIV, hepatitis B, and syphilis among pregnant women at the general hospital, Guatemala City, 20052009," Journal of the International Association of Physicians in AIDS Care, vol. 9, no. 5, pp. 313-317, 2010.

[31] C. S. Sebastiao, Z. Neto, D. Jandondo, M. Mirandela, J. Morais, and M. Brito, "HIV, hepatitis B virus, hepatitis $\mathrm{C}$ virus, and syphilis among pregnant women attending antenatal care in Luanda, Angola: seroprevalence and risk factors," Journal of Medical Virology, vol. 92, no. 12, pp. 3265-3270, 2020.

[32] H. Schumann, K. Rubagumya, J. Rubaihayo, G. Harms, R. K. Wanyenze, and S. Theuring, "The incidence of HIV and associated risk factors among pregnant women in Kabarole District, Uganda," PLoS One, vol. 15, no. 6, 2020.

[33] C. Agboghoroma and B. Ukaire, "Prevalence and risk factors of human immunodeficiency virus and hepatitis $\mathrm{C}$ virus infection among pregnant women attending antenatal care at a tertiary hospital in Abuja, Nigeria," Nigerian Medical Journal, vol. 61, no. 5, pp. 245-251, 2020.

[34] J. A. Ngaira, J. Kimotho, I. Mirigi et al., "Prevalence, awareness and risk factors associated with hepatitis B infection among pregnant women attending the antenatal clinic at Mbagathi District Hospital in Nairobi, Kenya," The Pan African Medical Journal, vol. 24, p. 315, 2016.

[35] S. Rashid, C. Kilewo, and S. Aboud, "Seroprevalence of hepatitis B virus infection among antenatal clinic attendees at a tertiary hospital in Dar es Salaam, Tanzania," Tanzania Journal of Health Research, vol. 16, no. 1, pp. 9-15, 2014.

[36] A. E. Woldegiorgis, W. Erku, G. Medhin, N. Berhe, and M. Legesse, "Community-based sero-prevalence of hepatitis $\mathrm{B}$ and $\mathrm{C}$ infections in south Omo zone, southern Ethiopia," PLoS One, vol. 14, no. 12, 2019.

[37] Y. Zenebe, W. Mulu, M. Yimer, and B. Abera, "Sero-prevalence and risk factors of hepatitis B virus and human immunodeficiency virus infection among pregnant women in Bahir Dar city, northwest Ethiopia: a cross sectional study," BMC Infectious Diseases, vol. 14, no. 1, p. 118, 2014.

[38] J. Blackard, J. Abesig, Y. Chen, H. Wang, F. M. Sompo, and I. X. Y. Wu, "Prevalence of viral hepatitis B in Ghana between 2015 and 2019: a systematic review and meta-analysis," PLoS One, vol. 15, no. 6, 2020.

[39] A. Getahun, M. Baekalia, N. Panda et al., "Seroprevalence of hepatitis B surface antigen in pregnant women attending antenatal clinic in Honiara Solomon Islands, 2015," World Journal of Hepatology, vol. 8, no. 34, pp. 1521-1528, 2016.
[40] A. Umare, B. Seyoum, T. Gobena, and T. Haile Mariyam, "Hepatitis B virus infections and associated factors among pregnant women attending antenatal Care Clinic at Deder Hospital, eastern Ethiopia," PLoS One, vol. 11, no. 11, 2016.

[41] T. Yohanes, Z. Zerdo, and N. Chufamo, "Seroprevalence and predictors of hepatitis B virus infection among pregnant women attending routine antenatal care in Arba Minch Hospital, south Ethiopia," Hepatitis Research and Treatment, vol. 2016, Article ID 9290163, 7 pages, 2016.

[42] A. Amsalu, G. Ferede, and D. Assegu, "High seroprevalence of syphilis infection among pregnant women in Yiregalem hospital southern Ethiopia," BMC Infectious Diseases, vol. 18, no. 1, p. 109, 2018.

[43] Y. Metaferia, W. Dessie, I. Ali, and A. Amsalu, "Seroprevalence and associated risk factors of hepatitis B virus among pregnant women in southern Ethiopia: a hospital-based cross-sectional study," Epidemiol Health, vol. 38, 2016.

[44] H. I. Mohamed, Z. M. Saad, E. M. Abd-Elreheem et al., "Hepatitis C, hepatitis B and HIV infection among Egyptian prisoners: seroprevalence, risk factors and related chronic liver diseases," Journal of Infection and Public Health, vol. 6, no. 3, pp. 186-195, 2013.

[45] K. Tareke, A. Munshea, and E. Nibret, "Seroprevalence of syphilis and its risk factors among pregnant women attending antenatal care at Felege Hiwot Referral Hospital, Bahir Dar, northwest Ethiopia: a cross-sectional study," BMC Research Notes, vol. 12, no. 1, p. 69, 2019.

[46] O. Ajayi, P. Nguku, I. Ajayi, and H. Dada-Adegbol, "Prevalence and risk factors of HIV and syphilis among pregnant women in Ado local government, Ekiti state, south western Nigeria, 2017," Journal of HIV \& Retro Virus, vol. 4, 2018.

[47] K. Genetu, Seroprevalence and Associated Factors of Human Immunodeficiency Virus, Hepatitis b Virus, and Syphilis among Pregnant Mothers Attending Antenatal Care at Governmental Hospitals in Addis Ababa, Ethiopia, [M.S. thesis], Addis Ababa University, Addis Ababa, 2021. 